\title{
Supramolecular Chemistry a Versatile Approach to Nanomaterial: A Review
}

\section{Keyur D Bhatt ${ }^{1 *}$, Ajay L Desai ${ }^{1}$ and Dashrath Kanzariya ${ }^{2}$}

${ }^{1}$ Department of Chemistry, Mehsana Urbane Institute of Sciences, Ganpat University, Kherva, India

2Department of Chemistry, SOT, PDPU, Gandhinagar, India

\section{Editorial \\ Volume 3 Issue 3}

Received Date: August 06, 2019

Published Date: August 26, 2019

DOI: $10.23880 /$ macij-16000143

*Corresponding author: Keyur D Bhatt, Department of Chemistry, Mehsana Urban Institute of Sciences, Ganpat University, Kherva-384012, Mehsana, Gujarat, India, Tel: 9825591630; Email: drkdbhatt@outllok.com

\section{Abstract}

Supramolecules are versatile drug receptors and finds application as nano medicines, drug sensors, medicinal chemistry, optical sensors, nonlinear optical materials, nano-capsule, nanoparticles, and paper based microfluidic device, supramolecular lectons, host molecules components in liquid crystals, photo resists, selective membranes surface reforming agents, anions and cation extraction agents. Fluoroionophores find application as chemosensor for ionic and neutral analytes by complexation and nanoparticles find their applications as chemosensor, nano biosensor and in drug delivery.

Keywords: Supramolecular Chemistry; Drug; Sensor; Drug Delivery System; Nano Material

\section{Introduction}

Nanotechnology may be defined as the design, characterization, evaluation, production and application of structures, devices and systems by controlling the size and shape at a nanometer scale. Nanoparticles in the size range of $\sim 1-100 \mathrm{~nm}$ offer new functions and applications in relation to bulk materials because of their unique electronic, magnetic, optical, mechanical, physical and chemical properties [1-3].

The last decade has seen many reports of chemical reduction routes for synthesis of nanoparticles using different reducing agents [4,5]. Supramolecules have received much attention in the recent past for their use as reducing as well as stabilising agent for the preparation of nanoparticles. Nanoparticles are the most extensively investigated nano materials due to their unique tunable optical properties, which can be applied in various applications such as sensing, detecting, and imaging [6-9] (Figure 1).

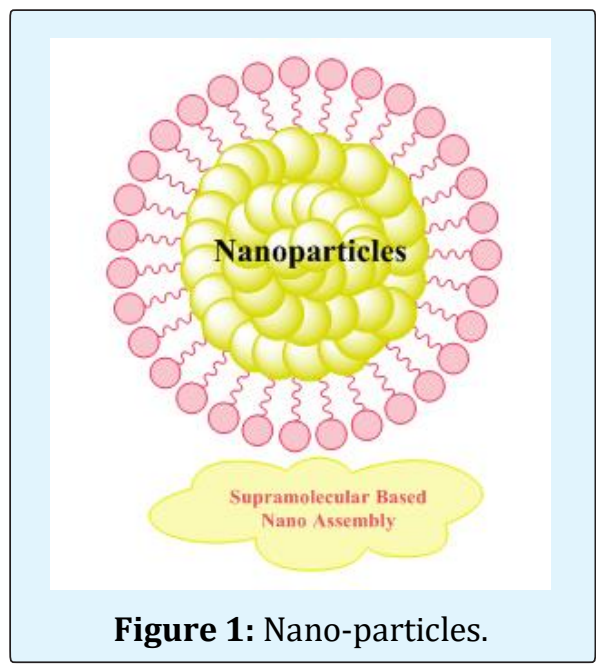

In recent years, there has been an increasing ecological/environmentally and global public health concern associated with environmental contamination by toxic metals ions [10]. Supramolecular chemistry has 


\section{Medicinal \& Analytical Chemistry International Journal}

been defined as the chemistry of molecular assemblies or expressed as the chemistry beyond the molecule or as the chemistry of non-covalent interactions [11] Supramolecular chemistry, recognized as the chemistry of molecular aggregates, is having a major impact on current chemical research, and will continue to do so into the foreseeable future. J.M. Lehn, D.J. Cram, and C.J. Pedersen were awarded Nobel Prize in chemistry in 1987 for pioneering work in the field of supramolecular chemistry. Based on the host-guest interactions and self-assembling process, a large fraction of supramolecular architectures are fabricated to perform functions and tasks. Different calix systems will be functionalized at 2 to 16 available sites with the moiety or functionality of our choice (e.g. amide, hydrazone, Schiff bases, azo group, sulphonic group, fluorophores etc.) by manipulating the reagent and reaction conditions [12].

It is of great interest to study the binding/interaction behavior of large number of the same or different functional groups into a calix system with various toxic cations, anions and neutral molecular ions by $\mathrm{pH}$ metry, spectrophotometry, spectrofluoremetry, colorimetry, and Mass and NMR titrations. Applications of these functionalized calix system will be explored for Fluorescent sensor, biosensor, metal ion sensor for environmental toxic ions [13]. Heavy and toxic ions are defined as metallic elements that have a relatively high density compared to water. With the statement that heaviness and toxicity are inter-related, heavy metals also include metalloids, such as Arsenic, Mercury, Zinc, Cadmium, Lead, Beryllium, Chromium etc. that are able to induce toxicity at low level of exposure. In recent years, there has been an increasing ecological/environmentally and global public health concern associated with environmental contamination by these metals [14].

Also, human revelation has risen dramatically as a result of an exponential increase of their use in several industrial, agricultural, domestic and technological applications. Reported sources of heavy metals in the environment include geogenic, industrial, agricultural, pharmaceutical, domestic effluents, and atmospheric sources [15]. Sensing applications of supramolecular chemistry are chosen for their binding size, shape and charge complementarity with the desired analysts, rely on exploiting the forces involved in the formation of noncovalent 'host-guest' complexes. Environmental pollution is very prominent in point source areas such as mining [16], foundries and smelters, and other metal-based industrial operations. Nano derived from supramolecules is one of the most advanced growing fields in chemistry.
Supramolecular chemistry has been stated that a field of inter-molecular chemistry based on the inherent covalent binding. Supramolecular chemistry is the host-guest interactions and ideal for nanomaterial because their modular character offers the binding with metal ions and shows applications in the field of physics, engineering and biosciences [17]. Supramolecules has an important advantage over nano science that they are readily obtainable with various substituents at the sites of the bridging methylene groups and various functionalizations on the hydroxy groups and amino group. It is of great interest to study when four/six/eight or more functional groups are present on one single molecule/macrocycle. Certainly it will exhibit some extra binding properties than those exhibited by a mono-functionalised hetrerocycles or benzene.

\section{- Reasons may be:}

More number of functional groups (donating and withdrawing groups), Functional groups may be circularly arranged/ pre organized, May acquire different conformation e.g. cone or partial cone, Enhanced/reduced binding due to hydrogen bonding, Hydrophobic nature of macrocycle may influence the binding, Due to intrinsic cavity/ hollow space and possibility of weak bonding in these macrocycles, may allow these compounds to behave as ditopic or tritopic receptors after functionalization they may form super structure by self-assembling and application in the field of Nanoscience's, Sensors, Dyes, Metal and DNA interactions etc [18-20].

Heavy and toxic metal ions are distinct as metallic elements that have a relatively high concentration compared to aqueous system. Heaviness and toxicity are inter-related; heavy metals also include metalloids, such as Arsenic, Mercury, Zinc, Cadmium, Lead, Beryllium, Chromium etc. that are able to induce toxicity at low level of exposure [21-23].

\section{Conclusion}

Review helps academic organization and research center in deep understanding the binding behavior of nanomaterial derived from supramolecules based device. As the kind of molecules, which are going to be synthesized find application not only as extractor and sensors but are also found to be biologically active and bio sensors. Therefore focus will also be on environmental safety pharmaceutical and chemical and food industries with a hope that microfluidic device may open new vista in advanced research. 


\section{Medicinal \& Analytical Chemistry International Journal}

\section{References}

1. Cushing BL, Kolesnichenko VL, O'Connor CJ (2004) Recent advances in the liquid-phase syntheses of inorganic nanoparticles. Chemical Reviews-Columbus 104(9): 3893-3946.

2. Tan Y, Dai X, Li Y, Zhu D (2003) Preparation of gold, platinum, palladium and silver nanoparticles by the reduction of their salts with a weak reductantpotassium bitartrate. Journal of Materials Chemistry 13(5): 1069-1075.

3. Schmid G (1992) Large clusters and colloids. Metals in the embryonic state. Chemical Reviews 92(8): 1709-1727.

4. Bhatt KD, Gupte HS, Makwana BA, Vyas DJ, Maity D, et al. (2012) Calix receptor edifice; scrupulous turn off fluorescent sensor for Fe (III), Co (II) and Cu (II). J Fluoresc 22(6): 1493-1500.

5. Makwana BA, Vyas DJ, Bhatt KD, Jain VK (2017) Selective sensing of copper (II) and leucine using fluorescent turn on-off mechanism from calix [4] resorcinarene modified gold nanoparticles. Sensors and Actuators B: Chemical 240: 278-287.

6. Templeton AC, Wuelfing WP, Murray RW (2000) Monolayer-protected cluster molecules. Accounts of Chemical Research 33(1): 27-36.

7. Grabar KC, Freeman RG, Hommer MB, Natan MJ (1995) Preparation and characterization of Au colloid monolayers. Anal Chem 67(4): 735-743.

8. Anshup JSV, Subramaniam C, Kumar RR, Priya S, Kumar TRS, et al. (2005) Growth of gold nanoparticles in human cells. Langmuir 21(25): 11562-11567.

9. Rouhana LL, Jaber JA, Schlenoff JB (2007) Aggregation-Resistant Water-Soluble Gold Nanoparticles, Langmuir 23(26): 12799-12801.

10. Balasubramanian R, Kim B, Tripp SL, Wang X, Lieberman M, et al. (2002) Dispersion and stability studies of resorcinarene-encapsulated gold nanoparticles. Langmuir 18(9): 3676-3681.

11. Guo Y, Wang Z, Shao H, Jiang X (2012) Stable fluorescent gold nanoparticles for detection of $\mathrm{Cu} 2+$ with good sensitivity and selectivity. Analyst 137(2): 301-304.
12. Upadhyayula VK (2012) Functionalized gold nanoparticle supported sensory mechanisms applied in detection of chemical and biological threat agents: a review. Anal Chim Acta 715: 1-18.

13. Tiwari PM, Vig K, Dennis VA, Singh SR (2011) Functionalized Gold Nanoparticles and Their Biomedical Applications. Nanomaterials 1(1): 31-63.

14. Omidfar K, Khorsand F, Azizi MD (2013) New analytical applications of gold nanoparticles as label in antibody based sensors. Biosensors and Bioelectronics 43: 336-347.

15. Bhatt KD, Makwana BA, Vyas DJ, Mishra DR, Jain VK (2014) Selective recognition by novel calix system: ICT based chemosensor for metal ions. Journal of Luminescence 146: 450-457.

16. Bhatt KD, Shah H, Modi KM, Kongor A, Panchal M, et al. (2017) Novel calix [4] pyrrole assembly: Punctilious recognition of $\mathrm{F}-$ and $\mathrm{Cu}+2$ ions. Journal of Molecular Structure 1149: 299-306.

17. Ghosh P, Han G, De M, Kim CK (2008) Rotello VM Gold nanoparticles in delivery applications. Advanced drug delivery reviews 60(11): 1307-1315.

18. Li B, Du Y, Dong S (2009) DNA based gold nanoparticles colorimetric sensors for sensitive and selective detection of $\mathrm{Ag}(\mathrm{I})$ ions. Anal Chim Acta 644(1-2): 78-82.

19. Tiwari NR (2010) Gold Nanoparticles for Colorimetric detection of hydrolysis of antibiotics by penicillin $G$ acylase. Advances in Bioscience and Biotechnology 1(4): 322-329.

20. Chen YC, Lee IL, Sung YM, Wu SP (2013) Triazole functionalized gold nanoparticles for colorimetric Cr3+ sensing. Sensors and Actuators B: Chemical 188: 354-359.

21. Bhatt KD, Shah HD, Modi KM, Narechania MB, Patel C (2019) Calix [4] pyrrole virtuous sensor: a selective and sensitive recognition for $\mathrm{Pb}$ (II) ions by spectroscopic and computational study. Supramolecular Chemistry 31(4): 268-282.

22. Makwana BA, Vyas DJ, Bhatt KD, Darji S, Jain VK (2016) Novel fluorescent silver nanoparticles: sensitive and selective turn off sensor for cadmium ions. Applied Nanoscience 6(4): 555-566. 


\section{Medicinal \& Analytical Chemistry International Journal}

23. Bhatt KD, Shah HD, Panchal M (2017) A switch-off fluorescence probe towards $\mathrm{Pb}$ (II) and cu (II) ions based on a calix [4] pyrrole bearing amino-quinoline group. Luminescence 32(8): 1398-1404. 